\title{
Derivation and validation of updated QFracture algorithm to predict risk of osteoporotic fracture in primary care in the United Kingdom: prospective open cohort study
}

\author{
(c) $\frac{(1)(8)}{\mathrm{gy}}$ MPEN ACCESS
}

\author{
Julia Hippisley-Cox professor of clinical epidemiology and general practice, Carol Coupland associate \\ professor of medical statistics
}

Division of Primary Care, University Park, Nottingham NG2 7RD, UK

\begin{abstract}
Objective To develop and validate an updated version of the QFracture algorithm for estimating the risk of a patient sustaining an osteoporotic fracture or hip fracture in a primary care population.

Design Prospective open cohort study using routinely collected data from 420 general practices in the United Kingdom to develop updated QFracture scores and 207 practices to validate scores. Cox's proportional hazards model was used in the derivation cohort to derive risk equations using several explanatory variables. We calculated measures of calibration and discrimination using the validation cohort.
\end{abstract}

Participants 3142673 patients in derivation cohort and 1583373 in validation cohort, aged 30-100 years, who contributed 23608337 and 11732106 person years of observation, respectively. We identified 59 772 incident diagnoses of osteoporotic fracture in the derivation cohort and 28685 in the validation cohort.

Outcomes Incident diagnosis of osteoporotic fracture (vertebral, distal radius, proximal humerus, or hip) and incident hip fracture recorded in general practice records or linked cause of death records.

Results We found significant independent associations with overall fracture risk in women for age, body mass index, ethnic origin, alcohol intake, smoking status, chronic obstructive pulmonary disease or asthma, any cancer, cardiovascular disease, dementia, diagnosis or treatment for epilepsy, history of falls, chronic liver disease, Parkinson's disease, rheumatoid arthritis or systemic lupus erythematosus, chronic renal disease, type 1 diabetes, type 2 diabetes, previous fracture, endocrine disorders, gastrointestinal malabsorption, any antidepressants, corticosteroids, unopposed hormone replacement therapy, and parental history of osteoporosis. Risk factors for hip fracture in women were similar except for gastrointestinal malabsorption and parental history of hip fracture. Risk factors for men were largely the same as those for women but also included care home residence. The updated hip fracture algorithm explained $71.7 \%$ (95\% confidence interval $71.1 \%$ to $72.3 \%$ ) of the variation in women and $70.4 \%(69.3 \%$ to $71.5 \%)$ in men. D statistic values for hip fracture were high for women (3.26, 3.21 to 3.31$)$ and men
(3.15, 3.06 to 3.24), and higher than for osteoporotic fracture. Values for the area under the receiver operating characteristics curves for hip fracture were 0.89 for women and 0.88 for men, compared with 0.79 and 0.71 for osteoporotic fracture, respectively. The updated algorithms performed better than the 2009 algorithms.

Conclusions Two QFracture algorithms were updated to predict risk of osteoporotic and hip fracture in primary care populations to include ethnic origin, all classes of antidepressants, chronic obstructive pulmonary disease, epilepsy, dementia, Parkinson's disease, cancer, systemic lupus erythematosus, chronic renal disease, type 1 diabetes, previous fragility fracture, and care home residence. These updated algorithms showed improved performance compared with previous QFracture algorithms reported in 2009.

\section{Introduction}

Osteoporotic fractures are a major and increasing cause of morbidity and a considerable burden to health services. Hip fractures in particular result in considerable pain, loss of function, and hospital care, making prevention a high priority for patients, physicians, and for public health. Therapeutic and lifestyle interventions exist that might reduce the risk of osteoporosis and hence a person's risk of fracture. ${ }^{1}$ The challenge now is to improve methods for accurate identification of people at high risk who might benefit most from a therapeutic or preventative intervention. Guideline ${ }^{2-6}$ recommend a targeted approach to the prevention of osteoporosis, based on the 10 year absolute risk of major osteoporotic fracture. Risk prediction tools are therefore needed to accurately estimate individual risk as well as enable a systematic targeted population based screening approach.

In 2009, we published a new risk prediction algorithm, QFracture. ${ }^{7}$ We designed the algorithm to estimate absolute risk of osteoporotic fracture and hip fracture in primary care. The algorithm is based on variables that are readily available in 
patients' electronic records in primary healthcare ${ }^{8}$ or that patients themselves would probably know without needing laboratory tests or clinical measurements. We designed this approach to enable the algorithms to be readily implemented in routine clinical practice or used by individual patients. The 2009 algorithms performed well when validated in a separate set of practices from the QResearch database ${ }^{7}$ and also performed well in a more stringent validation using a separate set of practices contributing to The Health Improvement Network database by an independent team. ${ }^{\text {? }}$

In February 2012, the National Institute for Health and Clinical Excellence (NICE) published draft guidance ${ }^{6}$ that recommended further developments to improve the use of QFracture, such as extending the age range to patients older than 85 years and including additional variables such as previous fragility fracture, ${ }^{6}$ ethnic group ${ }^{10}$ epilepsy and use of anticonvulsants, ${ }^{11}$ care home residency, ${ }^{12}{ }^{13}$ additional inflammatory arthropathies, chronic obstructive airways disease, ${ }^{6}$ type 1 diabetes, ${ }^{14}$ and other causes of immobility ${ }^{6}$ (such as Parkinson's disease ${ }^{15}$ or dementia).

We have therefore undertaken a study to update the original QFracture algorithms using the most recent version of the QResearch database and to test the performance of the updated algorithms in a separate set of practices from those used to develop the updated model. We also extended our original definition of osteoporotic fracture, which included hip, wrist, and vertebral fractures to include proximal humerus fractures that can also signify osteoporotic fractures. Since the methods, strengths, and limitations have already been published in detail both in the original paper ${ }^{7}$ and the independent validation, ${ }^{9}$ this article focuses on the changes to the algorithm.

\section{Methods}

\section{Study design and data source}

We conducted a prospective cohort study using a large population of patients in primary care from version 32 of the QResearch database (data last updated in October 2011).

QResearch is a large, validated, primary care electronic database containing the health records of more than 13 million patients registered from over 620 general practices using the Egton Medical Information System (EMIS) computer system (box 1). Practices and patients contained on the database are nationally representative for the United Kingdom and similar to those on other large, national, primary care databases using other clinical software systems. ${ }^{16}$ We included all QResearch practices once they had been using their current EMIS system for at least one year, to ensure completeness of recording of morbidity and prescribing data. We randomly allocated two thirds of practices to the derivation dataset and the remaining third to the validation dataset. $^{6}$

\section{Cohort selection}

We identified an open cohort of patients aged 30-100 years at the study entry date and registered with eligible practices at some time between 1 January 1993 and 1 October 2011. For each patient, we determined an entry date into the cohort, which was the latest of the following dates: patient's 30th birthday, date of registration with the practice plus one year, date on which the computer system was installed in the general practice plus one year, and beginning of the study period (1 January 1993). We only included patients in the analysis once they had a minimum of one year's complete data in their medical record. For each patient, we also determined an exit date, which was the earliest of the following dates: date of recorded fracture during follow-up, date of death, date of deregistration with the practice, date of last upload of computerised data, or the study end date (1 October 2011). Patients with a previous recorded fracture were eligible for inclusion in the cohort.

\section{Primary outcomes}

We had two primary outcomes: osteoporotic fracture defined as a diagnosis of a hip, vertebral, proximal humerus, or distal radius fracture during follow-up and diagnosis of hip fracture, where these fractures were recorded either on the general practice record or the linked death record. Unlike the 2009 QFracture algorithm, the definition of osteoporotic fracture also included fracture of the proximal humerus.

\section{Fracture risk factors}

We examined risk factors currently included in the 2009 QFracture algorithm. In addition to these factors, we also investigated other variables which have been associated with increased risk of osteoporosis and highlighted by NICE (box 2).

We restricted all values of these variables to those recorded in the person's electronic healthcare record before baseline, except for body mass index, alcohol intake, and smoking status. We used the values recorded closest to study entry date and recorded before the diagnosis of osteoporotic fracture (or for patients who did not develop a fracture, before censoring). We assumed that if there was no recorded value of a diagnosis, prescription, or family history, then the patient did not have that exposure.

\section{Model derivation and development}

We used Cox's proportional hazards models in the derivation dataset to estimate the coefficients and hazard ratios associated with each potential risk factor for recorded diagnosis of osteoporotic fracture and hip fracture for men and women separately using robust variance estimates to allow for the clustering of patients within general practices. We used a graphical method (complementary log-log plots) to check the assumption of proportional hazards. We used fractional polynomials to model non-linear risk associations with continuous variables if appropriate. ${ }^{32} \mathrm{We}$ used multiple imputation to replace missing values for alcohol intake, smoking status, and body mass index, and used these values in our main analyses. ${ }^{33-35} \mathrm{We}$ included all the potential predictor variables and the survival outcome terms in the imputation model. We used the imputation by chained equations procedure in Stata $^{36}$ to obtain five imputed datasets.

In view of the large number of variables under consideration and the need to ensure that the resulting algorithm can be used in everyday clinical practice, we explored whether any similar variables could be combined. We only considered combining variables that were similar clinically, based on decisions made before carrying out the analysis (for example, rheumatoid arthritis or systemic lupus erythematosus, tricyclic antidepressants or other antidepressants, and different types of hormone replacement therapy). We evaluated this by running a model with separate terms for each variable; if two similar variables were both significant (hazard ratio $<0.8$ or $>1.20$, and $\mathrm{P}<0.01$ ), we compared them with a direct significance test. If this comparison was not significant $($ at $\mathrm{P}<0.01)$ and if the hazard ratios were within 0.2 of each other, we combined the variables into a new variable (for example, either rheumatoid arthritis or systemic lupus erythematosus).

We fitted our final models on the basis of multiply imputed datasets using Rubin's rules to combine effect estimates and estimate standard errors to allow for the uncertainty due to 


\section{Box 1 QFracture web calculator}

A simple web calculator implements the updated QFracture algorithm is publicly available at www.qfracture.org. It also has the open source software for download

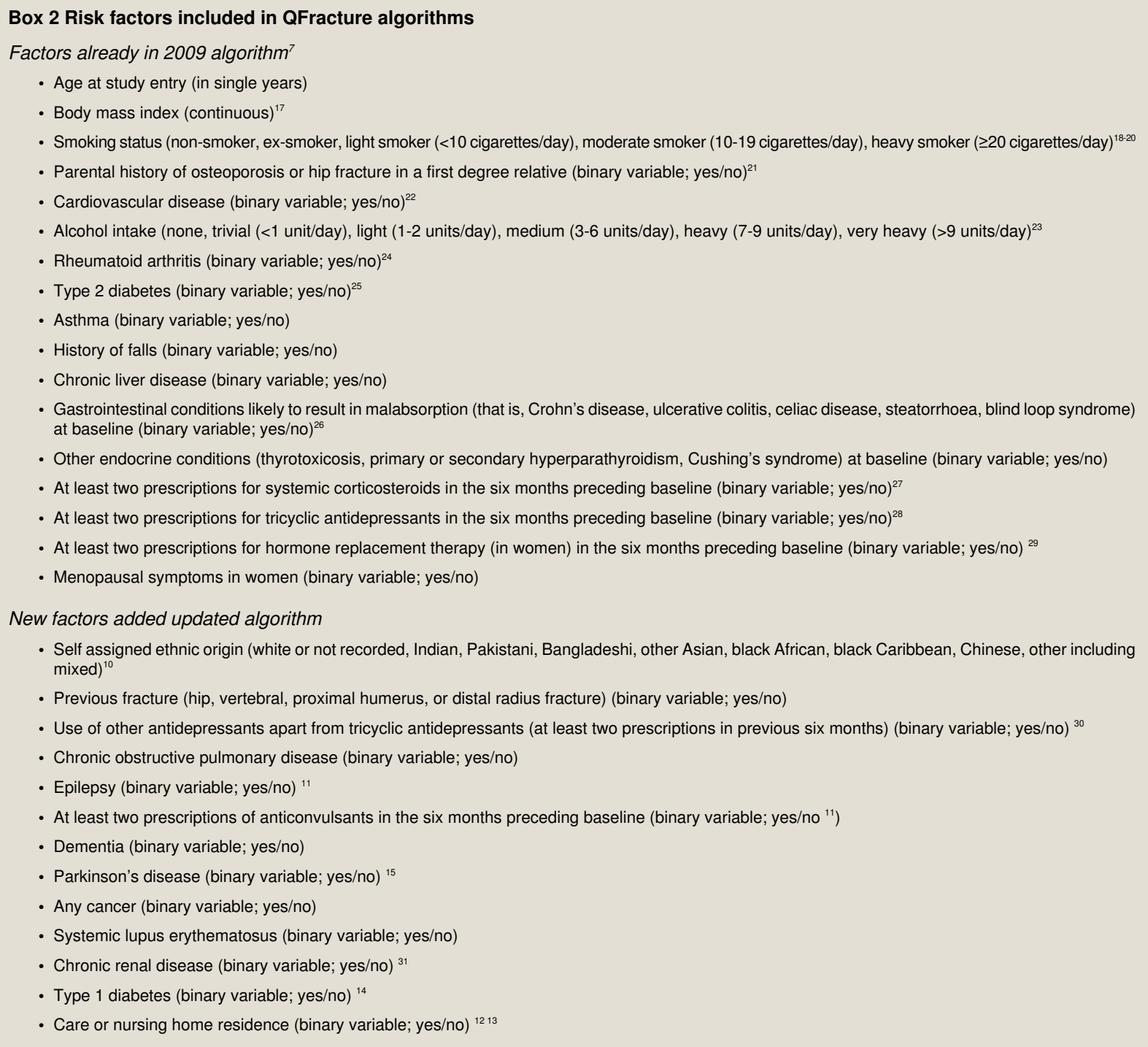

missing data. ${ }^{37}$ We took the regression coefficients (that is, log of the hazard ratios) from the final models and used them as weights. As in previous studies, ${ }^{38}{ }^{39}$ we combined these weights with the baseline survivor functions for osteoporotic fracture or hip fracture evaluated at 10 years to derive a risk equation for 10 years' follow-up. We also derived risk equations for each year from one to 15 years, so that users of the algorithm could select the time period over which fracture risk could be estimated.

\section{Validation of updated QFracture algorithm}

We tested the performances of the final models in the validation dataset. We calculated the estimated risks at 10 years of sustaining an osteoporotic fracture or hip fracture for each patient in the validation dataset using multiple imputation to replace missing values for alcohol intake, smoking status, and body mass index, as in the derivation dataset. We calculated the mean predicted fracture risk and the observed fracture risk at 10 years and compared these by every tenth of predicted risk. We calculated the D statistic (a measure of discrimination) ${ }^{40}$ and an $\mathrm{R}^{2}$ statistic (a measure of explained variation for survival data based on the D statistic). ${ }^{41} \mathrm{We}$ also calculated the area under the receiver operator characteristics curve at 10 years, in which higher values indicate better discrimination. We compared the performance of the updated scores with the original QFracture 2009 scores. We examined reclassification by defining high risk as the top tenth of predicted risk at 10 years for the original and updated QFracture scores. We then calculated the observed risk of fracture in patients who were reclassified from low to high or vice versa with updated algorithm, compared with the 2009 algorithm. We did not undertake a further comparison with the FRAX algorithm algorithms because these algorithms are not published or available from the authors.

We used all the available data from the QResearch database and therefore did not do a pre-study sample size calculation. There were at least 100 events per variable in the prediction modelling for both outcomes in the derivation cohort. ${ }^{42}$ All analyses were conducted using Stata (version 11). 


\section{Results \\ Characteristics of derivation and validation datasets}

Of 627 UK practices meeting our inclusion criteria, 420 were randomly assigned to the derivation dataset and 207 to the validation dataset. Table $1 \Downarrow$ compares the key characteristics of eligible patients in each cohort. The derivation cohort had 3 142673 patients, of whom 59628 had a recorded previous fracture, either of the hip (9621 patients), distal radius (40 305), vertebra (7412), or proximal humerus (2290) based on the first previous fracture recorded. The validation cohort had 1583373 patients, of whom 27907 had a previous fracture of the hip (4233), distal radius (19 212), vertebra (3438), or proximal humerus (1024). Although this validation cohort was drawn from an independent group of general practices, the baseline characteristics were very similar to those for the derivation cohort.

\section{Incidence rates by ethnic origin}

Table $2 \Downarrow$ shows the crude and age standardised incidence rates in each cohort by ethnic group. Overall in the derivation cohort, 59772 patients had incident osteoporotic fractures arising from 23608337 person years during follow-up, resulting in a crude incidence rate of 253 per 100000 person years $(95 \%$ confidence interval 251 to 255). These incident fractures included 26726 $(44.7 \%)$ distal radius fractures, 20028 (33.5\%) hip fractures, $9236(15.5 \%)$ vertebral fractures, and $3782(6.3 \%)$ proximal humerus fractures based on the first fracture recorded during follow-up. Of the 59772 incident osteoporotic fractures, 45476 $(76.1 \%)$ occurred in women and $3760(6.3 \%)$ in patients older than 85 years. Of 20028 hip fractures in the derivation cohort, 14864 occurred in women. In the validation cohort, 28685 patients had incident osteoporotic fractures, of which 21677 $(75.6 \%)$ were women. In the validation cohort, we identified 9610 hip fractures, 7089 of which occurred in women.

\section{Model development}

Tables $3 \Downarrow$ and $4 \Downarrow$ show results of the multivariate final Cox regression analysis for the fracture outcomes in women and men, respectively. Figure $1 \Downarrow$ shows the adjusted hazard ratios for the updated fractional polynomial terms for age and body mass index, for both outcomes in men and women.

\section{Risk factors for fracture in women}

After including the other variables in the model, we found significant associations with osteoporotic fracture risk in women for the following variables: age, body mass index, ethnic origin, alcohol intake, smoking status, chronic obstructive airways disease or asthma, any cancer, cardiovascular disease, dementia, diagnosis or treatment for epilepsy, history of falls, chronic liver disease, Parkinson's disease, rheumatoid arthritis or systemic lupus erythematosus, chronic renal disease, type 1 diabetes, type 2 diabetes, previous fracture, endocrine disorders, gastrointestinal malabsorption, any antidepressants, corticosteroids, unopposed hormone replacement therapy, and parental history of osteoporosis (table 3). The risk factors for hip fracture were similar, except gastrointestinal malabsorption and parental history of osteoporosis were not significant.

\section{Risk factors for fracture in men}

We found significant associations with osteoporotic fracture risk in men for the variables shown in table 4 . The list of variables was the same as for women, although care home residency was significant in men only and endocrine problems were not significant in men. All the factors that were significant predictors for osteoporotic fracture in men were also significant factors for hip fracture, except for gastrointestinal malabsorption.

\section{Ethnic origin}

Ethnic origin was strongly associated with fracture risk in men and women. In women, the risk of osteoporotic fracture was significantly lower for the other ethnic groups compared with the reference category of white women (which also included patients whose ethnic origin was unrecorded; table 3). The pattern was similar for hip fracture in women and for both outcomes in men (table 4).

\section{Type 1 and type 2 diabetes}

Type 1 and type 2 diabetes were significantly associated with both osteoporotic fracture and hip fracture in men and women, with higher risks of these outcomes for type 1 diabetes than type 2 diabetes. Type 1 diabetes was associated with a substantially higher risk of hip fracture in women (adjusted hazard ratio 4.63 (95\% confidence interval 3.35 to 6.39 )) than the risk of osteoporotic fracture (1.92 (1.55 to 2.37)). In men, the adjusted hazard ratio for hip fracture associated with type 1 diabetes was 4.83 (3.25 to 71.7 ) compared with 2.33 (1.83 to 2.96) for osteoporotic fracture.

\section{Antidepressants}

All types of antidepressants (tricyclic antidepressants, selective serotonin reuptake inhibitors, and others) were associated with increased fracture risks in both men and women, which were similar in magnitude for each type of antidepressant. We therefore combined these variables into one to indicate current treatment with antidepressants. In women, antidepressants were associated with increased risks of osteoporotic fracture (adjusted hazard ratio 1.37 (95\% confidence interval 1.33 to 1.42 ) and hip fracture (1.39 (1.33 to 1.46); table 3 ). The adjusted hazard ratios were marginally higher in men than in women, with adjusted hazard ratios of 1.60 (1.50 to 1.70) for osteoporotic fracture and 1.69 (1.53 to 1.86) for hip fracture (table 4).

\section{Other risk factors included in updated QFracture model}

The updated algorithms also included chronic obstructive airways disease, chronic renal disease cancer, dementia, epilepsy (either diagnosed or prescribed anticonvulsants), Parkinson's disease, chronic renal disease, previous fragility fracture, and care home residency (men only). Men who were residents in care homes had an increased risk of both hip fracture (adjusted hazard ratio 2.05 (95\% confidence interval 1.43 to 2.93$)$ ) and osteoporotic fracture (1.59 (1.14 to 2.22); table 4).

\section{Validation of the updated QFracture algorithms}

Table $5 \Downarrow$ shows the discrimination statistics for the updated QFracture algorithms in the validation cohort. The hip fracture algorithm had the best performance, explaining 71.7\% (95\% confidence interval $71.1 \%$ to $72.3 \%$ ) of the variation in women and $70.4 \%(69.3 \%$ to $71.5 \%)$ in men. D statistic values were 3.26 (3.21 to 3.31) for women and (3.15, 3.06 to 3.24) for men. The values for the area under the receiver operator characteristics curve for hip fracture were 0.89 for women and 0.88 for men, compared with 0.79 and 0.71 for osteoporotic fracture, respectively. 
Performance statistics for the updated algorithms were better than those for the 2009 algorithms (table 5). For example, for hip fracture in women, the updated algorithm explained $71.7 \%$ of the variation, compared with $63.9 \%$ for the 2009 algorithm. Discrimination was also significantly higher for the updated algorithms, with a D statistic of 3.26 for hip fracture in women compared with 2.73 for the 2009 algorithm. We repeated the analyses and restricted the sample to patients aged $30-85$ years, and the performance statistics were still better for the updated algorithms than for the 2009 algorithms (results not shown).

Figure $2 \Downarrow$ shows the observed and predicted risks at 10 years for osteoporotic fracture, using the updated algorithm. Overall, the calibration was good with a small over prediction in the highest tenth of risk. We obtained similar results for hip fracture (fig $3 \Downarrow$ ).

\section{Thresholds and sensitivity}

Table $6 \Downarrow$ shows the cut-off values for the top tenth of risk based on 10 year predicted risk for hip fracture and osteoporotic fracture in the validation cohort. A cut-off value of $5.3 \%$ would identify $3295(59.8 \%)$ of the 5509 women with a hip fracture over the next 10 years. Corresponding values for hip fracture in men were $1.3 \%$ for the cut-off value and $64.3 \%$ for sensitivity. The sensitivity for hip fracture using the updated algorithm was higher for both men and women than that using the 2009 algorithm (that is, $52.7 \%$ for women and $54.2 \%$ for men). The sensitivity values were also higher when evaluated over five years; for example, $71 \%$ of hip fractures over five years in both men and women were in the top tenth of predicted risks.

\section{Clinical example 1}

A 64 year old white woman is a light smoker, has type 1 diabetes, epilepsy, and a body mass index of 22 . She has a 10 year risk of $15 \%$ for osteoporotic fracture (that is, hip, distal radius, vertebra or proximal humerus) and a 13\% risk of hip fracture. A woman with the same characteristics who is Pakistani would have a corresponding 10 year risk of osteoporotic fracture of $7 \%$ and hip fracture of $6 \%$.

\section{Clinical example 2}

A 70 year old white man with a body mass index of 22 is a heavy smoker, and has cancer and a history of falls. He has a 10 year risk of osteoporotic fracture of $8 \%$ and a $6 \%$ risk of hip fracture. A man with the same characteristics who is also taking antidepressants will have a risk of $12 \%$ for osteoporotic fracture and $9 \%$ for hip fracture. If he also lives in a care home, his risks are $19 \%$ for osteoporotic fracture and $18 \%$ for hip fracture.

\section{Clinical example 3}

A 88 year old white man with a body mass index of 22 is an ex-smoker, has chronic renal disease, and is taking antidepressants and steroid tablets. He has a five year risk of osteoporotic fracture of $12 \%$ and a five year risk of hip fracture of $9 \%$. If he was not taking antidepressants, his five year risk of osteoporotic fracture would be $7 \%$ and his risk of hip fracture would be $6 \%$.

\section{Discussion}

We have presented a substantial upgrade to the QFracture algorithms, designed to better identify patients at increased risk of hip and osteoporotic fracture so that they can be targeted for interventions to reduce their fracture risk. Our study deals with recommendations made by the recent draft guidance from NICE on the assessment of fracture risk. ${ }^{6}$ There are four main changes to the algorithms. Firstly, the age range over which the algorithms can be applied is extended to include the most elderly patients (that is, $>85$ years). Secondly, the algorithm expands the definition of osteoporotic fracture to include fracture of the proximal humerus. Thirdly, it also includes a broader range of predictor variables such as ethnic origin, use of other antidepressants apart from tricyclic antidepressants, chronic obstructive pulmonary disease, epilepsy, dementia, Parkinson's disease, cancer, systemic lupus erythematosus, chronic renal disease, type 1 diabetes, previous fragility fracture, and care home residence. Finally, the algorithm simplifies the variable of hormone replacement therapy by combining 12 categories to one for unopposed oestrogen, which is the predominant effect.

The algorithms can derive five year absolute risks, which could be more useful than 10 year absolute risks in very elderly patients whose mean life expectancy is less than 10 years. ${ }^{43}$ The shorter time period also might indicate the duration over which bisphosphonates might be prescribed, in view of the lack of long term safety data. The web based calculator, however, can be used to calculate risks over any time period between one and 15 years (box 1 ).

Currently, QRISK2 ${ }^{38}$ (www.qrisk.org) is fully integrated into most clinical computer systems in UK general practices, and QFracture can be implemented in a similar way. For example, alerts can be set to identify patients suitable for risk assessment, which informs the doctor before seeing the patient. A structured template can pop up for the doctor to complete, which already includes information from the patients' records. The doctor then adds any new information to calculate the score, which is then displayed graphically for discussion with the patient. The results can be posted into the patients' records. Alternatively, batch processing tools could be used, which calculate an estimated risk for all eligible patients registered with the practice. This list can then be sorted so that patients with the highest risk can be recalled.

\section{New predictors-comparison with other studies}

To our knowledge, this is the first algorithm for fracture risk prediction to account for different risks by ethnic group in both men and women in the UK. This inclusion is important, since minority ethnic groups are expected to make up to $20 \%$ of the UK's population by 2051 and such tools for fracture risk prediction need to take account of this diversity ${ }^{6}$ and current evidence of disparities in screening for and treating osteoporosis between ethnic groups. ${ }^{10}$ The reduced risk of fracture for the non-white ethnic groups is comparable with that reported in a study of postmenopausal women in the United States. ${ }^{25}$

We found that the risk of osteoporotic fracture increased among patients diagnosed with epilepsy as well as those prescribed anticonvulsants. The magnitude and direction of this increased risk is comparable to the increased risk associated with anticonvulsants recently reported in a Canadian population based case-control study. ${ }^{11}$ We also found an increased risk of fracture for men and women associated with use of selective serotonin reuptake inhibitors and other antidepressants- the magnitude and direction of the increased risk was broadly similar between the different types of antidepressants in our study and similar to results published elsewhere. ${ }^{30}$ Similarly, the increased fracture risk among patients with Parkinson's disease in our study is comparable to findings from a recent matched cohort study of incident cases of Parkinson's disease ${ }^{15}$ and the risk associated with chronic renal disease accorded with 
previous studies. ${ }^{31}$ We recorded a four to five fold increase in the risk of hip fracture associated with type 1 diabetes, which was similar to the risk reported in a Swedish cohort study. ${ }^{44}$ The risks for type 1 diabetes are substantially higher than the two fold increase associated with type 2 diabetes, both in our study or reported elsewhere. ${ }^{25}$ Our study focused on predictor variables and was not designed to evaluate the mechanisms by which risk factors mediate their effect. The role of hypoglycaemic drugs in fracture risk, however, deserves more detailed study. Lastly, we found a raised risk of fracture among men living in care homes that corresponded with increased risks reported elsewhere. $^{12} 13$

\section{Methodological issues}

Although the updated QFracture algorithm is more complex than its original version, it has improved discrimination and sensitivity. The advantages of using routinely collected data to develop risk prediction scores are that they can be updated to reflect changes in populations, improvements in data quality, advances in knowledge regarding relevant predictor variables and evolving requirements (such as new guidelines or quality standards). The resulting algorithms can also be implemented in the primary care setting since the data are already present. Since QFracture has been designed to be integrated into clinical computer systems, much of the apparent complexity relating to additional variables can be incorporated into the software using data already entered into the patient's electronic health record.

The methods to derive and validate this model are the same as those used for the original development of QFracture and a range of other risk prediction tools. The strengths and limitations of the approach have already been discussed in detail. ${ }^{7} 91645-47$ The present validation was done in a separate set of practices to those that were used to develop the score, although the practices all use the same clinical computer system (EMIS, used by $55 \%$ of UK general practitioners). A more stringent validation by an independent team using data from another clinical system used in the UK (Vision, used by $20 \%$ of general practitioners) is planned and will be reported in due course. However, the existing independent external validation of QFracture ${ }^{9}$ showed comparable, if not marginally improved, performance compared with the validation on the QResearch database. ${ }^{7}$

In summary, we have updated two algorithms to predict risk of osteoporotic fracture and hip fracture in a primary care population. The updated algorithms include additional terms for ethnic group and several risk factors, and can be applied to a wider age range. They show improved performance compared with previous QFracture algorithms. Further research should evaluate the clinical outcomes and cost effectiveness of these algorithms in primary care.

\section{We thank EMIS and the general practices using EMIS for their} contributions to the QResearch database.

Contributors: JH-C designed the study, obtained approvals, reviewed the literature, prepared the data, developed and tested the algorithms, undertook the primary analysis and interpretation, and wrote the first and subsequent draft manuscript. CC contributed to the analysis, development and testing of the algorithms, interpretation, and drafting of the manuscript. $\mathrm{JH}-\mathrm{C}$ is the guarantor.

Funding: The study received no external funding.

Competing interests: All authors have completed the Unified Competing Interest form at www.icmje.org/coi_disclosure.pdf (available on request from the corresponding author) and declare: no support from any organisation for the submitted work; $\mathrm{JH}-\mathrm{C}$ is a professor of clinical epidemiology at the University of Nottingham and codirector of
QResearch, a not-for-profit organisation that is a joint partnership between the University of Nottingham and EMIS (commercial IT supplier for $60 \%$ of general practices in the $\mathrm{UK}$ ); $\mathrm{JH}-\mathrm{C}$ is also a paid director of ClinRisk Limited, which produces open and closed source software to ensure the reliable and updatable implementation of clinical risk algorithms within clinical computer systems to help improve patient care; $\mathrm{CC}$ is an associate professor of medical statistics at the University of Nottingham and a paid consultant statistician for ClinRisk Limited; no other relationships or activities that could appear to have influenced the submitted work.

Ethical approval: The study was approved by the QResearch Scientific board and was approved by the Trent research ethics committee.

Data sharing: The algorithms presented in this study will be released as open source software under the GNU Lesser General Public Licence version 3. The open source software allows use by anyone without charge under the terms of the GNU Lesser Public License version 3. Closed source software can be licensed at a fee.

1 National Institute for Health and Clinical Excellence. Systematic review of clinical effectiveness prepared for the guideline: "Osteoporosis assessment of fracture risk and the prevention of osteoporotic fractures in individuals at high risk", 2008: 205.

2 Kanis JA, Burlet N, Cooper C, Delmas PD, Reginster JY, Borgstrom F, et al. European guidance for the diagnosis and management of osteoporosis in postmenopausal women. Osteoporos Int 2008;19:399-428.

3 Dawson-Hughes B, Tosteson A, Melton L, Baim S, Favus M, Khosla S, et al. Implications of absolute fracture risk assessment for osteoporosis practice guidelines in the USA. Osteoporosis Int 2008:19:449-58.

4 Kanis JA, McCloskey EV, Johansson H, Strom O, Borgstrom F, Oden A. Case finding for the management of osteoporosis with FRAX-assessment and intervention thresholds for the UK. Osteoporos Int 2008;19:1395-408.

5 National Osteoporosis Guideline Group. Guideline for the diagnosis and management of osteoporosis in postmenopausal men and women from the age of 50 in the UK. 2008 : 12.

6 National Institute for Health and Clinical Excellence. Osteoporosis: fragility fracture risk. Short clinical guideline-evidence and recommendation. National Clinical Guideline Centre, 2012: 90.

7 Hippisley-Cox J, Coupland C. Predicting risk of osteoporotic fracture in men and women in England and Wales: prospective derivation and validation of QFractureScores. BMJ 2009;339:b4229.

8 Kush RD, Helton E, Rockhold FW, Hardison CD. Electronic health records, medical research, and the Tower of Babel. N Engl J Med 2008;358:1738-40.

9 Collins GS, Mallett S, Altman DG. Predicting risk of osteoporotic and hip fracture in the United Kingdom: prospective independent and external validation of QFractureScores. BMJ 2011;342:d3651.

10 Cauley J. Defining ethnic and racial differences in osteoporosis and fragility fractures. Clin Orthop Relat Res 2011;469:1891-9.

11 Jette N, Lix LM, Metge CJ, Prior HJ, McChesney J, Leslie WD. Association of antiepileptic drugs with nontraumatic fractures: a population-based analysis. Arch Neurol 2011;68:107-12.

12 Brennan nee Saunders J, Johansen A, Butler J, Stone M, Richmond P, Jones S, et al. Place of residence and risk of fracture in older people: a population-based study of over 65-year-olds in Cardiff. Osteoporos Int 2003;14:515-9.

13 Godden S, Pollock AM. The use of acute hospital services by elderly residents of nursing and residential care homes. Health Soc Care Community 2001;9:367-74.

14 Janghorbani M, Van Dam RM, Willett WC, Hu FB. Systematic review of type 1 and type 2 diabetes mellitus and risk of fracture. Am J Epidemiol 2007:166:495-505

15 Chen Y-Y, Cheng P-Y, Wu S-L, Lai C-H. Parkinson's disease and risk of hip fracture: an 8-year follow-up study in Taiwan. Parkinsonism Relat Disord 2012, doi:10.1016/..parkreldis. 2012.01.014.

16 Hippisley-Cox J, Coupland C, Vinogradova Y, Robson J, Brindle P. Performance of the QRISK cardiovascular risk prediction algorithm in an independent UK sample of patients from general practice: a validation study. Heart 2008;94:34-39.

17 De Laet C, Kanis JA, Oden A, Johanson H, Johnell O, Delmas P, et al. Body mass index as a predictor of fracture risk: a meta-analysis. Osteoporos Int 2005;16:1330-8.

18 Vestergaard P, Mosekilde L. Fracture risk associated with smoking: a meta-analysis. $J$ Intern Med 2003;254:572-83.

19 Cornuz J, Feskanich D, Willett WC, Colditz GA. Smoking, smoking cessation, and risk of hip fracture in women. Am J Med 1999;106:311-4.

20 Hollenbach KA, Barrett-Connor E, Edelstein SL, Holbrook T. Cigarette smoking and bone mineral density in older men and women. Am J Public Health 1993:83:1265-70.

21 Diaz MN, O'Neill TW, Silman AJ. The influence of family history of hip fracture on the risk of vertebral deformity in men and women: the European Vertebral Osteoporosis Study. Bone 1997;20:145-9.

22 Kanis $\mathrm{J}$, Oden $\mathrm{A}$, Johnell $\mathrm{O}$. Acute and long-term increase in fracture risk after hospitalization for stroke. Stroke 2001;32:702-6.

23 Kanis JA, Johansson H, Johnell O, Oden A, De Laet C, Eisman JA, et al. Alcohol intake as a risk factor for fracture. Osteoporos Int 2005;16:737-42.

24 Spector TD, Hall GM, McCloskey EV, Kanis JA. Risk of vertebral fracture in women with rheumatoid arthritis. BMJ 1993;306:558.

25 Robbins J, Aragaki AK, Kooperberg C, Watts N, Wactawski-Wende J, Jackson RD, et al. Factors associated with 5-year risk of hip fracture in postmenopausal women. JAMA 2007;298:2389-98.

26 Kanis JA, Johnell O, Oden A, Johansson H, McCloskey E. FRAX and the assessment of fracture probability in men and women from the UK. Osteoporos Int 2008;19:385-97.

27 van Staa T, Leufkens H, Abenhaim L, Zhang B, Copper C. Use of oral corticosteroids and risk of fractures. J Bone Miner Res 2000;15:993-1000. 


\section{What is already known on this topic}

Osteoporotic fracture is a major cause of morbidity and interventions exist that can help reduce risk of fracture

Several international guidelines suggest a targeted approach for identifying high risk patients likely to benefit from interventions, based on a 10 year absolute risk of fracture

\section{What this study adds}

We have updated the QFracture algorithm to predict risk of hip fracture and osteoporotic fracture

The updated algorithm improved on previous algorithms by including ethnic origin, prescription of any class of antidepressants, chronic obstructive pulmonary disease, epilepsy, dementia, Parkinson's disease, cancer, systemic lupus erythematosus, chronic renal disease, type 1 diabetes, previous fragility fracture, and care home residence

Validation statistics suggest that the updated algorithm is probably more effective than previous algorithms at identifying patients at high risk of fracture in primary care in the UK

28 Hubbard R, Farrington P, Smith C, Smeeth L, Tattersfield A. Exposure to tricyclic and selective serotonin reuptake inhibitor antidepressants and the risk of hip fracture. $A m \mathrm{~J}$ Epidemiol 2003;158:77-84.

29 Michaelsson K, Baron JA, Farahmand BY, Johnell O, Magnusson C, Persson PG, et al. Hormone replacement therapy and risk of hip fracture: population based case-control study. The Swedish Hip Fracture Study Group. BMJ 1998;316:1858-63.

30 Coupland C, Dhiman P, Morriss R, Arthur A, Barton G, Hippisley-Cox J. Antidepressant use and risk of adverse outcomes in older people: population based cohort study. BMJ 2011;343:d4551

31 Nitsch D, Mylne A, Roderick PJ, Smeeth L, Hubbard R, Fletcher A. Chronic kidney disease and hip fracture-related mortality in older people in the UK. Nephrol Dial Transplant 2009;24:1539-44

32 Royston P, Ambler G, Sauerbrei W. The use of fractional polynomials to model continuous risk variables in epidemiology. Int J Epidemiol 1999;28:964-74.

33 Schafer J, Graham J. Missing data: our view of the state of the art. Psychol Methods 2002;7:147-77.

34 Steyerberg EW, van Veen M. Imputation is beneficial for handling missing data in predictive models. J Epidemiol Community Health 2007;60:979.

35 Moons KGM, Donders RART, Stijnen T, Harrell FJ. Using the outcome for imputation of missing predictor values was preferred. J Epidemiol Community Health 2006;59:1092.

36 Royston P. Multiple imputation of missing values: update of ice. Stata J 2005;5:527-36.

37 Royston P. Multiple imputation of missing values. Stata J 2004;4:227-41.

38 Hippisley-Cox J, Coupland C, Vinogradova Y, Robson J, Minhas R, Sheikh A, et al. Predicting cardiovascular risk in England and Wales: prospective derivation and validation of QRISK2. BMJ 2008;336:1475

39 Hippisley-Cox J, Coupland C, Vinogradova Y, Robson J, May M, Brindle P. Derivation and validation of QRISK, a new cardiovascular disease risk score for the United Kingdom: prospective open cohort study. BMJ 2007:335:136.
40 Royston P, Sauerbrei W. A new measure of prognostic separation in survival data. Stat Med 2004;23:723-48.

41 Royston P. Explained variation for survival models. Stata J 2006;6:1-14

42 Steyerberg E. Clinical prediction models. Springer, 2009.

43 McCann M, O'Reilly D, Cardwell C. A census-based longitudinal study of variations in survival amongst residents of nursing and residential homes in Northern Ireland. Age Ageing 2009;38:711-7.

44 Miao J, Brismar K, Nyrén O, Ugarph-Morawski A, Ye W. Elevated hip fracture risk in type 1 diabetic patients. Diabetes Care 2005;28:2850-5.

45 Hippisley-Cox J, Coupland C, Robson J, Sheikh A, Brindle P. Predicting risk of type 2 diabetes in England and Wales: prospective derivation and validation of QDScore. BMJ 2009;338:b880.

46 Collins GS, Altman DG. External validation of the QDScore for predicting the 10-year risk of developing type 2 diabetes. Diabetic Med 2011;28:599-607.

47 Hippisley-Cox J, Coupland C. Development and validation of risk prediction algorithm (QThrombosis) to estimate future risk of venous thromboembolism: prospective cohort study. BMJ 2011;343:d4656.

Accepted: 09 May 2012

\section{Cite this as: BMJ 2012;344:e3427}

This is an open-access article distributed under the terms of the Creative Commons Attribution Non-commercial License, which permits use, distribution, and reproduction in any medium, provided the original work is properly cited, the use is non commercial and is otherwise in compliance with the license. See: http://creativecommons.org/licenses/by$\mathrm{nc} / 2.0 /$ and http://creativecommons.org/licenses/by-nc/2.0/legalcode. 


\title{
Tables
}

\begin{abstract}
Table 1 | Characteristics of patients aged 30-100 years at baseline in derivation and validation cohorts, 1993-2011. Data are no (\%) of patients
\end{abstract} unless indicated otherwise

\begin{tabular}{|c|c|c|}
\hline & Derivation cohort $(n=3142673)$ & Validation cohort ( $n=1583$ 373) \\
\hline Female & $1598294(50.9)$ & $804563(50.8)$ \\
\hline Male & $1544379(49.1)$ & $778810(49.2)$ \\
\hline \multicolumn{3}{|l|}{ Age } \\
\hline Mean age (standard deviation) & $50(16)$ & $50(16)$ \\
\hline 30-39 years & $1046694(33.3)$ & $551004(34.8)$ \\
\hline $40-49$ years & $697053(22.2)$ & $345936(21.8)$ \\
\hline $50-59$ years & $530823(16.9)$ & $256353(16.2)$ \\
\hline $60-69$ years & $395846(12.6)$ & $194261(12.3)$ \\
\hline $70-79$ years & $288004(9.2)$ & $144152(9.1)$ \\
\hline $80-89$ years & $155893(5.0)$ & $77794(4.9)$ \\
\hline $90+$ years & $28360(0.9)$ & $13873(0.9)$ \\
\hline \multicolumn{3}{|l|}{ Body mass index } \\
\hline With recorded body mass index & $2358735(75.1)$ & $1164895(73.6)$ \\
\hline Mean (standard deviation) & $26.2(4.6)$ & $26.1(4.6)$ \\
\hline \multicolumn{3}{|l|}{ Smoking status } \\
\hline Non-smoker & $1554900(49.5)$ & $773198(48.8)$ \\
\hline Ex-smoker & $521244(16.6)$ & 257087 (16.2) \\
\hline Current smoking amount not recorded & $119012(3.8)$ & $65106(4.1)$ \\
\hline Current light smoker & $182124(5.8)$ & $94400(6.0)$ \\
\hline Current moderate smoker & $226074(7.2)$ & $113757(7.2)$ \\
\hline Current heavy smoker & $177535(5.6)$ & $86787(5.5)$ \\
\hline Not recorded & $361784(11.5)$ & $193038(12.2)$ \\
\hline \multicolumn{3}{|l|}{ Self assigned ethnic origin } \\
\hline Ethnic origin recorded & $1401264(44.6)$ & $727888(46.0)$ \\
\hline White or not recorded & $2995462(95.3)$ & $1493455(94.3)$ \\
\hline Indian & $28882(0.9)$ & $17670(1.1)$ \\
\hline Pakistani & $15364(0.5)$ & $6489(0.4)$ \\
\hline Bangladeshi & $9445(0.3)$ & $4191(0.3)$ \\
\hline Other Asian & $16902(0.5)$ & $10779(0.7)$ \\
\hline Caribbean & $14959(0.5)$ & $10144(0.6)$ \\
\hline Black African & $25210(0.8)$ & $17367(1.1)$ \\
\hline Chinese & $7727(0.2)$ & $5206(0.3)$ \\
\hline Other & $28722(0.9)$ & $18072(1.1)$ \\
\hline \multicolumn{3}{|l|}{ Alcohol intake } \\
\hline Non-drinker & $644877(20.5)$ & 330695 (20.9) \\
\hline Trivial (<1 unit/day) & 832859 (26.5) & $402847(25.4)$ \\
\hline Light (1-2 units/day) & $592441(18.9)$ & $287441(18.2)$ \\
\hline Moderate (3-6 units/day) & $172648(5.5)$ & $84478(5.3)$ \\
\hline Heavy (7-9 units/day) & $17895(0.6)$ & $8743(0.6)$ \\
\hline Very heavy ( $>9$ units/day) & $15688(0.5)$ & $7429(0.5)$ \\
\hline Alcohol consumption not recorded & $866265(27.6)$ & $461740(29.2)$ \\
\hline \multicolumn{3}{|l|}{ Medical and social factors } \\
\hline Previous fracture & $59628(1.9)$ & $27907(1.8)$ \\
\hline Dementia & $17289(0.6)$ & $7791(0.5)$ \\
\hline History of falls & $37656(1.2)$ & $17382(1.1)$ \\
\hline
\end{tabular}


Table 1 (continued)

\begin{tabular}{|c|c|c|}
\hline & Derivation cohort $(n=3142673)$ & Validation cohort ( $n=1583373$ ) \\
\hline Malabsorption & $17012(0.5)$ & $8026(0.5)$ \\
\hline Endocrine disorders & $16407(0.5)$ & $7882(0.5)$ \\
\hline Asthma & 210181 (6.7) & $99512(6.3)$ \\
\hline Chronic obstructive airways disease & $41893(1.3)$ & $19895(1.3)$ \\
\hline Asthma or chronic obstructive airways disease & $238185(7.6)$ & $113175(7.1)$ \\
\hline Any cancer & $59296(1.9)$ & $28203(1.8)$ \\
\hline Cardiovascular disease & $166600(5.3)$ & $77824(4.9)$ \\
\hline Epilepsy diagnosis & $32766(1.0)$ & $15442(1.0)$ \\
\hline Prescribed anticonvulsants & $42487(1.4)$ & $19745(1.2)$ \\
\hline Epilepsy diagnosis or prescribed anticonvulsants & $55859(1.8)$ & $26271(1.7)$ \\
\hline Chronic liver disease & $6119(0.2)$ & $3216(0.2)$ \\
\hline Parkinson's disease & $7809(0.2)$ & $3650(0.2)$ \\
\hline Rheumatoid arthritis & $21308(0.7)$ & $9350(0.6)$ \\
\hline Systemic lupus erythematosus & $1627(0.1)$ & $827(0.1)$ \\
\hline Rheumatoid arthritis or systemic lupus erythematosus & $22785(0.7)$ & $10091(0.6)$ \\
\hline Chronic renal disease & $6948(0.2)$ & $3413(0.2)$ \\
\hline Type 1 diabetes & $8997(0.3)$ & $4322(0.3)$ \\
\hline Type 2 diabetes & $88540(2.8)$ & $43437(2.7)$ \\
\hline Parental history of osteoporosis & $8716(0.3)$ & $4227(0.3)$ \\
\hline Resident in care or nursing home & $6303(0.2)$ & $1535(0.1)$ \\
\hline \multicolumn{3}{|l|}{ Current drug treatment } \\
\hline Corticosteroids & $69023(2.2)$ & $30998(2.0)$ \\
\hline Unopposed hormone replacement therapy & $33858(1.1)$ & $14988(0.9)$ \\
\hline \multicolumn{3}{|l|}{ Antidepressants } \\
\hline Selective serotonin reuptake inhibitors & $123486(3.9)$ & $55080(3.5)$ \\
\hline Tricyclic antidepressants & $122268(3.9)$ & $56779(3.6)$ \\
\hline Other & $19947(0.6)$ & $9976(0.6)$ \\
\hline Any & $241399(7.7)$ & $111229(7.0)$ \\
\hline
\end{tabular}


Table 2| Incidence of osteoporotic fracture (hip, distal radius, vertebra, or proximal humerus), person years of observation, and crude and age standardised rates of incidence, by ethnic origin of patient

\begin{tabular}{|c|c|c|c|c|c|c|c|c|}
\hline & \multicolumn{4}{|c|}{ Derivation cohort } & \multicolumn{4}{|c|}{ Validation cohort } \\
\hline & $\begin{array}{c}\text { Total person } \\
\text { years of } \\
\text { observation }\end{array}$ & $\begin{array}{c}\text { No of } \\
\text { patients } \\
\text { with new } \\
\text { fractures }\end{array}$ & $\begin{array}{c}\text { Crude fracture rate } \\
\text { per } 100000 \text { person } \\
\text { years }(95 \% \mathrm{Cl})\end{array}$ & $\begin{array}{l}\text { Age standardised } \\
\text { rate per } 100000 \\
\text { person years }(95 \% \\
\mathrm{Cl})\end{array}$ & $\begin{array}{c}\text { Total person } \\
\text { years of } \\
\text { observation }\end{array}$ & $\begin{array}{c}\text { No of } \\
\text { patients } \\
\text { with new } \\
\text { fractures }\end{array}$ & $\begin{array}{c}\text { Crude fracture rate } \\
\text { per } 100000 \text { person } \\
\text { years }(95 \% \mathrm{Cl})\end{array}$ & $\begin{array}{c}\text { Age standardised } \\
\text { rate per } 100000 \\
\text { person years }(95 \% \\
\mathrm{Cl})\end{array}$ \\
\hline $\begin{array}{l}\text { White or not } \\
\text { recorded }\end{array}$ & 22850547 & 59175 & 259 (257 to 261 ) & 255 (253 to 257 ) & 11253010 & 28318 & 252 (249 to 255 ) & 247 (244 to 250 ) \\
\hline Indian & 173614 & 212 & 122 (107 to 140$)$ & 152 (129 to 176$)$ & 105356 & 134 & $127(107$ to 151$)$ & 183 (146 to 219$)$ \\
\hline Pakistani & 82657 & 64 & 77 (61 to 99) & 94 (69 to 120$)$ & 38578 & 34 & 88 (63 to 123$)$ & 106 (66 to 147$)$ \\
\hline Bangladeshi & 46980 & 24 & 51 (34 to 76 ) & 76 (40 to 112$)$ & 26960 & 11 & 41 (23 to 74 ) & 49 (19 to 78$)$ \\
\hline Other Asian & 72453 & 53 & 73 (56 to 96$)$ & 145 (83 to 206 ) & 47326 & 29 & 61 (43 to 88 ) & 125 (58 to 192$)$ \\
\hline Caribbean & 110255 & 52 & 47 (36 to 62 ) & 61 (41 to 81$)$ & 75524 & 35 & 46 (33 to 65$)$ & 53 (35 to 72) \\
\hline Black African & 98740 & 43 & 44 (32 to 59$)$ & 77 (49 to 105$)$ & 72589 & 44 & 61 (45 to 81$)$ & 112 (62 to 163$)$ \\
\hline Chinese & 36895 & 37 & 100 (73 to 138$)$ & 200 (111 to 290$)$ & 23956 & 18 & 75 (47 to 119$)$ & 170 (61 to 280$)$ \\
\hline $\begin{array}{l}\text { Other ethnic } \\
\text { group }\end{array}$ & 136195 & 112 & 82 (68 to 99) & 133 (101 to 165$)$ & 88807 & 62 & 70 (54 to 90$)$ & $122(86$ to 159$)$ \\
\hline Overall total & 23608337 & 59772 & 253 (251 to 255 ) & - & 11732106 & 28685 & 245 (242 to 247 ) & - \\
\hline
\end{tabular}


Table 3| Multivariate analysis for osteoporotic fracture and hip fracture in women in the derivation cohort. Data are adjusted hazard ratios (95\% confidence interval)

Osteoporotic fracture

Ethnic origin

White or not recorded

Indian

Pakistani

Bangladeshi

Other Asian

Black Caribbean

Black African

Chinese

Other ethnic group

\section{Alcohol intake}

Non-drinker

Trivial (<1 unit/day)

Light (1-2 units/day)

Moderate (3-6 units/day)

Heavy (7-9 units/day)

Very heavy ( $>9$ units/day)

Smoking status

Non-smoker

Ex-smoker

Current light smoker

Current moderate smoker

Current heavy smoker

Medical or social factors*

Asthma or chronic obstructive airways disease

Any cancer

Cardiovascular disease

Dementia

Epilepsy diagnosis or prescribed anticonvulsants

History of falls

Chronic liver disease

Parkinson's disease

Rheumatoid arthritis or systemic lupus erythematosus

Chronic renal disease

Type 1 diabetes

Type 2 diabetes

Previous fracture

Endocrine disorders

Gastrointestinal malabsorption

Parental history of osteoporosis

Any antidepressants

Corticosteroids

Unopposed hormone replacement therapy

0.85 ( 0.80 to 0.91 )
1

0.75 (0.61 to 0.92$)$

0.46 (0.32 to 0.65 )

0.44 (0.27 to 0.72 )

0.56 (0.41 to 0.76 )

0.23 (0.15 to 0.33 )

0.48 (0.34 to 0.69 )

0.61 (0.40 to 0.90$)$

0.64 (0.51 to 0.79 )

1

1.00 (0.98 to 1.02$)$

1.05 (1.02 to 1.09 )

1.18 (1.09 to 1.27$)$

1.61 (1.27 to 2.04 )

1.87 (1.47 to 2.39 )

Hip fracture

1

0.60 (0.41 to 0.86$)$

0.46 (0.24 to 0.88 )

0.56 (0.22 to 1.43$)$

0.58 (0.29 to 1.16$)$

0.27 (0.15 to 0.47 )

0.10 (0.01 to 0.68 )

0.35 (0.14 to 0.92 )

0.49 (0.30 to 0.80$)$

\section{1}

0.88 (0.85 to 0.92 )

0.91 (0.86 to 0.95 )

1.06 (0.92 to 1.22$)$

1.56 (0.97 to 2.51$)$

1.94 (1.31 to 2.87 )

\section{1}

1.04 (1.01 to 1.07 )

1

1.10 (1.05 to 1.15$)$

1.08 (1.04 to 1.13$)$

1.13 (1.08 to 1.18$)$

1.33 (1.24 to 1.42 )

1.17 (1.12 to 1.23$)$

1.37 (1.27 to 1.47 )

1.62 (1.49 to 1.76$)$

1.27 (1.23 to 1.31$)$

1.23 (1.16 to 1.31$)$

1.27 (1.20 to 1.34 )

1.31 (1.22 to 1.42 )

1.21 (1.17 to 1.26$)$

1.22 (1.17 to 1.29 )

1.97 (1.80 to 2.13 )

2.57 (2.31 to 2.85 )

1.54 (1.45 to 1.63 )

1.62 (1.48 to 1.76 )

1.57 (1.47 to 1.68 )

1.54 (1.42 to 1.68 )

1.89 (1.60 to 2.24 )

1.91 (1.45 to 2.51 )

1.64 (1.47 to 1.83 )

2.03 (1.75 to 2.35 )

1.33 (1.25 to 1.43 )

1.69 (1.53 to 1.86$)$

1.27 (1.07 to 1.51 )

1.51 (1.17 to 1.96 )

1.92 (1.55 to 2.37)

4.63 (3.35 to 6.39 )

1.27 (1.21 to 1.34$)$

1.57 (1.45 to 1.69 )

1.08 (1.03 to 1.13 )

1.73 (1.62 to 1.85 )

1.23 (1.14 to 1.34 )

1.33 (1.19 to 1.50$)$

1.17 (1.06 to 1.29$)$

Not significant

1.74 (1.47 to 2.05 )

Not significant

1.37 (1.33 to 1.42 )

1.39 (1.33 to 1.46 )

1.21 (1.15 to 1.27$)$

Models also included fractional polynomial terms for age and body mass index. Hazard ratios simultaneously adjusted for all the other variables in the table as well as fractional polynomial terms for age and body mass index. Fractional polynomial terms were (age/10) $)^{2},(\text { age } / 10)^{3}$, (body mass index/10) $)^{-1}$ for osteoporotic fracture; and $(\text { age } / 10)^{2},(\text { age } / 10)^{3}$, and (body mass index $\left./ 10\right)^{-2}$ for hip fracture.

${ }^{*}$ Compared with patients without the condition/medication at baseline except for type 1 and type 2 diabetes, which were compared with no diabetes. 
Table 4| Multivariate analysis for osteoporotic fracture and hip fracture in men in the derivation cohort. Data are adjusted hazard ratios (95\% confidence interval)

Osteoporotic fracture

\section{Ethnic origin}

White or not recorded

Indian

Pakistani

Bangladeshi

Other Asian

Black Caribbean

Black African

Chinese

Other ethnic group

\section{Alcohol intake}

Non-drinker

Trivial ( $<1$ unit/day)

Light (1-2 units/day)

Moderate (3-6 units/day)

Heavy (7-9 units/day)

Very heavy (>9 units/day)

Smoking status

Non-smoker

Ex-smoker

Current light smoker

1.00 (0.95 to 1.05$)$

0.93 (0.88 to 0.98$)$

1.00 (0.96 to 1.06$)$

$1.12(1.04$ to 1.20$)$

$1.32(1.16$ to 1.51$)$

Current moderate smoker

$1.17(1.10$ to 1.24$)$

Current heavy smoker

$1.29(1.21$ to 1.37$)$

1.32 (1.24 to 1.41$)$

Medical and social factors ${ }^{\star}$

Asthma or chronic obstructive airways disease

1.33 (1.26 to 1.42$)$

Any cancer

$1.60(1.43$ to 1.73$)$

Cardiovascular disease

1.26 (1.19 to 1.34$)$

Dementia

Epilepsy diagnosis or prescribed anticonvulsants

1.90 (1.58 to 2.28$)$

2.19 (2.00 to 2.39$)$

History of falls

Chronic liver disease

$1.72(1.51$ to 1.96$)$

Parkinson's disease

2.58 (2.01 to 3.32$)$

2.45 (2.06 to 2.92 )

Rheumatoid arthritis or systemic lupus erythematosus

1.55 (1.33 to 1.82$)$

Chronic renal disease

Type 1 diabetes

Type 2 diabetes

Previous fracture

Gastrointestinal malabsorption

Care or nursing home resident

Parental history of osteoporosis

Any antidepressants

Corticosteroids
1.58 (1.20 to 2.08 )

2.33 (1.83 to 2.96 )

1.25 (1.15 to 1.36$)$

1.25 (1.03 to 1.51$)$

1.59 (1.14 to 2.22$)$

5.47 (3.41 to 8.80$)$

1.60 (1.50 to 1.70$)$

1.34 (1.23 to 1.47$)$
1.35 (1.20 to 1.53$)$

\section{Hip fracture}

1

0.62 (0.36 to 1.08$)$

0.64 (0.38 to 1.10$)$

0.13 (0.02 to 0.92 )

0.41 ( 0.13 to 1.29$)$

0.22 (0.08 to 0.59 )

0.89 (0.40 to 1.99$)$

0.46 (0.12 to 1.82$)$

$0.80(0.45$ to 1.42$)$

Models also included fractional polynomial terms for age and body mass index. Hazard ratios simultaneously adjusted for all the other variables in the table as well as fractional polynomial terms for age and body mass index. Fractional polynomial terms were $(\text { age } / 10)^{0.5},(\text { body mass index } / 10)^{-1}$, and $(\text { body mass index } / 10)^{-0.5}$ for osteoporotic fracture; and $(\text { age/10 })^{2},(\text { age/10) })^{3}$ and (body mass index $\left./ 10\right)^{-2}$ for hip fracture.

${ }^{*}$ Compared with patients without the condition/medication at baseline except for type 1 and type 2 diabetes, which were compared with no diabetes. 
Table 5| Validation statistics* of QFracture algorithms for osteoporotic fracture and hip fracture in validation cohort. Data are mean (95\% confidence interval)

\begin{tabular}{|c|c|c|c|c|}
\hline \multirow[b]{2}{*}{ Statistic } & \multicolumn{2}{|c|}{ Osteoporotic fracture } & \multicolumn{2}{|c|}{ Hip fracture } \\
\hline & Age $30-85$ years, 2009 algorithm & $\begin{array}{c}\text { Age } 30-100 \text { years, updated } \\
\text { algorithm }\end{array}$ & Age $30-85$ years, 2009 algorithm & $\begin{array}{c}\text { Age 30-100 years, updated } \\
\text { algorithm }\end{array}$ \\
\hline \multicolumn{5}{|l|}{ Women } \\
\hline $\mathrm{R}^{2}$ & 44.87 (43.07 to 46.67$)$ & 51.9 (51.2 to 52.6$)$ & $63.94(62.12$ to 65.76$)$ & $71.73(71.10$ to 72.30$)$ \\
\hline D & 1.85 (1.78 to 1.91$)$ & 2.13 (2.10 to 2.15$)$ & 2.73 (2.62 to 2.83 ) & 3.26 (3.21 to 3.31 ) \\
\hline $\mathrm{ROC}$ & $0.788(0.786$ to 0.790$)$ & 0.790 (0.787 to 0.793$)$ & 0.890 (0.889 to 0.892$)$ & 0.893 (0.890 to 0.896$)$ \\
\hline \multicolumn{5}{|l|}{ Men } \\
\hline $\mathrm{R}^{2}$ & 30.02 (22.21 to 37.84$)$ & 38.20 (36.89 to 39.57$)$ & 63.19 (60.81 to 65.57$)$ & 70.37 (69.25 to 71.49$)$ \\
\hline $\mathrm{D}$ & 1.34 (1.09 to 1.59$)$ & 1.61 (1.56 to 1.66$)$ & 2.68 (2.55 to 2.82 ) & 3.15 (3.06 to 3.24 ) \\
\hline $\mathrm{ROC}$ & $0.688(0.684$ to 0.692$)$ & $0.711(0.703$ to 0.719$)$ & $0.856(0.851$ to 0.860$)$ & 0.875 (0.868 to 0.883$)$ \\
\hline
\end{tabular}

ROC=receiver operator characteristics.

${ }^{*}$ Higher values indicate better discrimination. 
Table 6| Number of cases of osteoporotic fracture and hip fracture over 10 years in the top tenth of risk in the validation cohort, based on 10 year predicted risk

\begin{tabular}{|c|c|c|c|c|}
\hline Outcome & $\begin{array}{l}\text { Cut-off value for top tenth of predicted } \\
\text { risk (\%) }\end{array}$ & $\begin{array}{l}\text { Fractures in top tenth of predicted } \\
\text { risk (no) }\end{array}$ & $\begin{array}{l}\text { Total fractures in } 10 \text { year time period } \\
\text { (no) }\end{array}$ & Sensitivity (\%) \\
\hline \multicolumn{5}{|l|}{ Women } \\
\hline Hip fracture & 5.3 & 3295 & 5509 & 59.8 \\
\hline Osteoporotic fracture & 11.1 & 5321 & 15275 & 34.8 \\
\hline \multicolumn{5}{|l|}{ Men } \\
\hline Hip fracture & 1.3 & 636 & 1739 & 64.3 \\
\hline Osteoporotic fracture & 2.6 & 1793 & 4827 & 37.1 \\
\hline
\end{tabular}




\section{Figures}

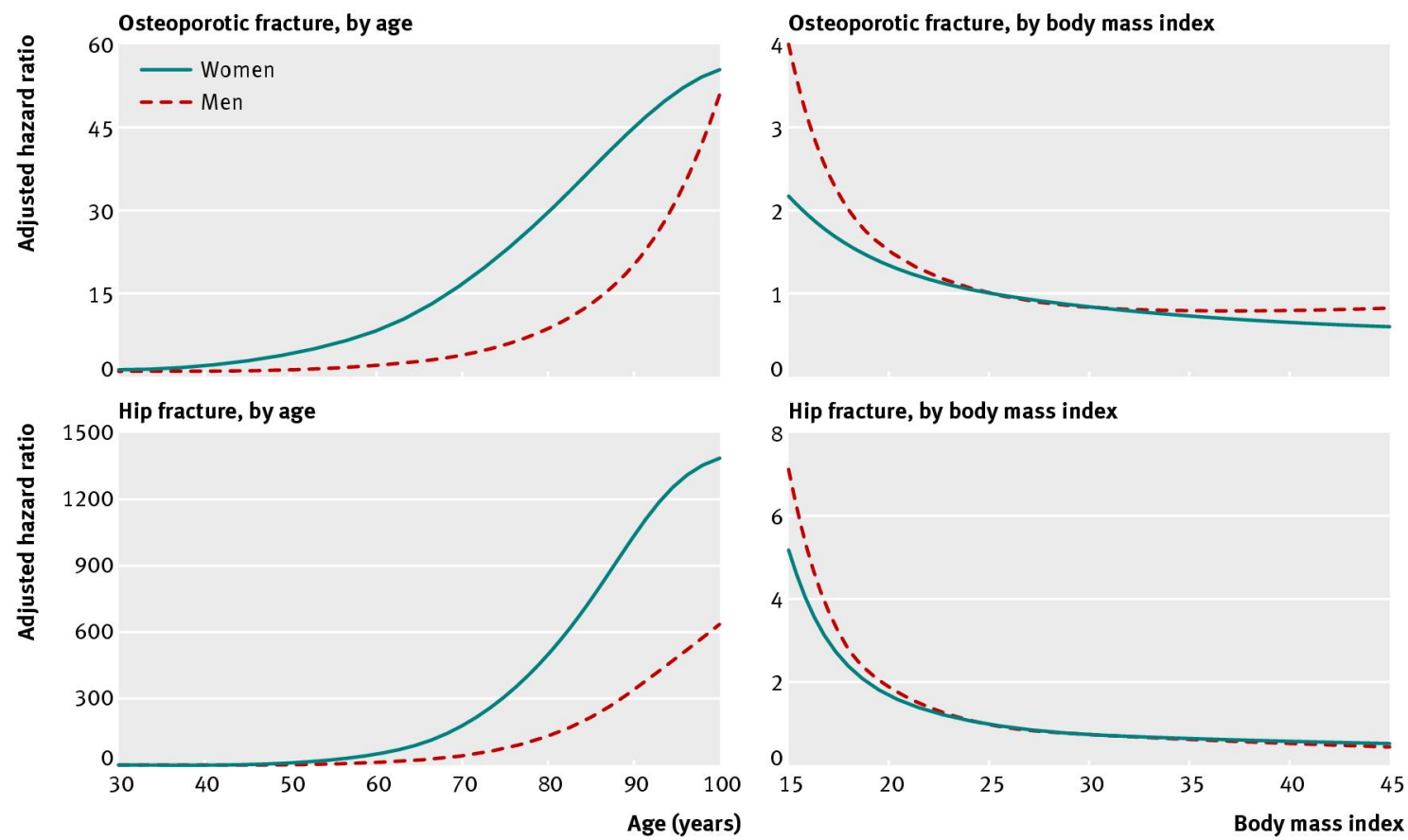

Fig 2 Predicted and observed risks of osteoporotic fracture in the validation cohort using updated QFracture algorithm, over 10 years 


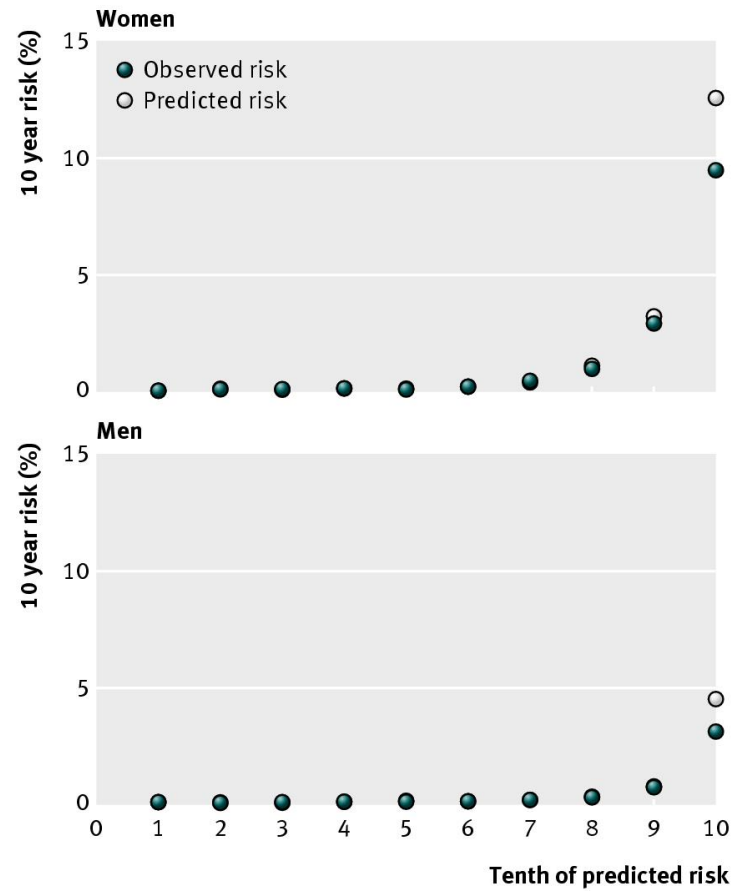

Fig 3 Predicted and observed risks of hip fracture in the validation cohort using updated QFracture algorithm, over 10 years 